\title{
Nonlocal Problem for Fractional Evolution Equations of Mixed Type with the Measure of Noncompactness
}

\author{
Pengyu Chen and Yongxiang Li \\ Department of Mathematics, Northwest Normal University, Lanzhou 730070, China \\ Correspondence should be addressed to Pengyu Chen; chpengyu123@163.com
}

Received 8 December 2012; Revised 14 March 2013; Accepted 18 March 2013

Academic Editor: Xinan Hao

Copyright (C) 2013 P. Chen and Y. Li. This is an open access article distributed under the Creative Commons Attribution License, which permits unrestricted use, distribution, and reproduction in any medium, provided the original work is properly cited.

\begin{abstract}
A general class of semilinear fractional evolution equations of mixed type with nonlocal conditions on infinite dimensional Banach spaces is concerned. Under more general conditions, the existence of mild solutions and positive mild solutions is obtained by utilizing a new estimation technique of the measure of noncompactness and a new fixed point theorem with respect to convexpower condensing operator.
\end{abstract}

\section{Introduction}

In this paper, we use a new estimation technique of the measure of noncompactness and fixed point theorem with respect to convex-power condensing operator to discuss the existence of mild solutions and positive mild solutions for nonlocal problem of fractional evolution equations (NPFEE) of mixed type with noncompact semigroup in Banach space E:

$$
\begin{aligned}
{ }^{C} D_{t}^{q} u(t)+A u(t)= & f(t, u(t), G u(t), S u(t)), \quad t \in J, \\
& u(0)=g(u),
\end{aligned}
$$

where ${ }^{C} D_{t}^{q}$ is the Caputo fractional derivative of order $q$; $0<$ $q<1, A: D(A) \subset E \rightarrow E$ is a closed linear operator and $-A$ generates a uniformly bounded $C_{0}$-semigroup $T(t)(t \geq 0)$ in $E, f: J \times E \times E \times E \rightarrow E$ is a Carathéodory type function, $J=[0, a], a>0$ is a constant, $g$ mapping from some space of functions to be specified later, and

$$
\begin{array}{ll}
G u(t)=\int_{0}^{t} K(t, s) u(s) d s, & t \in J, \\
S u(t)=\int_{0}^{a} H(t, s) u(s) d s, & t \in J,
\end{array}
$$

are integral operators with integral kernels $K \in C(\Delta, \mathbb{R})$, $\Delta=\{(t, s) \mid 0 \leq s \leq t \leq a\}$, and $H \in C\left(\Delta_{0}, \mathbb{R}\right), \Delta_{0}=\{(t, s) \mid$ $0 \leq t, s \leq a\}$.

In recent years, fractional calculus has attracted many physicists, mathematicians, and engineers, and notable contributions have been made to both theory and applications of fractional differential equations. It has been found that the differential equations involving fractional derivatives in time are more realistic to describe many phenomena in practical cases than those of integer order in time. For more details about fractional calculus and fractional differential equations we refer to the books by Miller and Ross [1], Podlubny [2], and Kilbas et al. [3] and the papers by Eidelman and Kochubei [4], Lakshmikantham and Vatsala [5], Agarwal et al. [6], Darwish and Ntouyas [7-10], and Darwish et al. [11]. One of the branches of fractional calculus is the theory of fractional evolution equations. Since fractional order semilinear evolution equations are abstract formulations for many problems arising in engineering and physics, fractional evolution equations have attracted increasing attention in recent years; see [12-26] and the references therein.

The study of abstract nonlocal Cauchy problem was initiated by Byszewski and Lakshmikantham [27]. Since it is demonstrated that the nonlocal problems have better effects in applications than the traditional Cauchy problems, differential equations with nonlocal conditions were studied 
by many authors and some basic results on nonlocal problems have been obtained; see [17-32] and the references therein for more comments and citations. In the past few years, the existence, uniqueness, and some other properties of mild solutions to nonlocal problem of fractional evolution equations (1) with $f(t, u(t), G u(t), S u(t))=f(t, u(t))$ have been extensively studied by using Banach contraction mapping principal, Schauder's fixed point theorem and Krasnoselskii's fixed point theorem, when $T(t)(t \geq 0)$ is a compact semigroup. For more details on the basic theory of nonlocal problem for fractional evolution equations, one can see the papers of Diagana et al. [17], Wang et al. [18], Li et al. [19], Zhou and Jiao [20], Wang et al. [21], Wang et al. [22], Wang et al. [23], Chang et al. [24], Balachandran and Park [25], and Balachandran and Trujillo [26]. However, for the case that the semigroup $T(t)(t \geq 0)$ is noncompact, there are very few papers studied nonlocal problem of fractional evolution equations; that only Wang et al. [14] discussed the existence of mild solutions for nonlocal problem of fractional evolution equations under the situation that $T(t)(t \geq 0)$ is an analytic semigroup of uniformly bounded linear operators.

It is well known that the famous Sadovskii's fixed point theorem is an important tool to study various differential equations and integral equations on infinite dimensional Banach spaces. Early on, Lakshmikantham and Leela [33] studied the following initial value problem (IVP) of ordinary differential equation in Banach space $E$ :

$$
\begin{gathered}
u^{\prime}(t)=f(t, u(t)), \quad t \in J, \\
u(0)=u_{0},
\end{gathered}
$$

and they proved that if for any $R>0, f$ is uniformly continuous on $J \times B_{R}$ and satisfies the noncompactness measure condition

$$
\alpha(f(t, D)) \leq L \alpha(D), \quad \forall t \in J, D \subset B_{R},
$$

where $B_{R}=\{u \in E:\|u\| \leq R\}, L$ is a positive constant, $\alpha(\cdot)$ denotes the Kuratowski measure of noncompactness in $E$, then IVP (3) has a global solution provided that $L$ satisfies the condition

$$
L<\frac{1}{a}
$$

In fact, there are a large amount of authors who studied ordinary differential equations in Banach spaces similar to (3) by using Sadovskii's fixed point theorem and hypothesis analogous to (4); they also required that the constants satisfy a strong inequality similar to (5). For more details on this fact, we refer to Guo [34], Liu et al. [35] and Liu et al. [36].

It is easy to see that the inequality (5) is a strong restrictive condition, and it is difficult to be satisfied in applications. In order to remove the strong restriction on the constant $L$, Sun and Zhang [37] generalized the definition of condensing operator to convex-power condensing operator. And based on the definition of this new kind of operator, they established a new fixed point theorem with respect to convexpower condensing operator which generalizes the famous Schauder's fixed point theorem and Sadovskii's fixed point theorem. As an application, they investigated the existence of global mild solutions and positive mild solutions for the initial value problem of evolution equations in $E$ :

$$
\begin{gathered}
u^{\prime}(t)+A u(t)=f(t, u(t)), \quad t \in J, \\
u(0)=u_{0} ;
\end{gathered}
$$

they assume that $-A$ generate a equicontinuous $C_{0^{-}}$semigroup; the nonlinear term $f$ is uniformly continuous on $J \times B_{R}$ and satisfies a suitable noncompactness measure condition similar to (4). Recently, Shi et al. [38] developed the IVP (6) to the case that the nonlinear term is $f(t, u(t), G u(t), S u(t))$ and obtained the existence of global mild solutions and positive mild solutions by using the new fixed point theorem with respect to convex-power condensing operator established in [37], but they also require that the nonlinear term $f$ is uniformly continuous on $J \times B_{R} \times B_{R} \times B_{R}$.

We observed that, in [33-38], the authors all demand that the nonlinear term $f$ is uniformly continuous; this is a very strong assumption. As a matter of fact, if $f(t, u)$ is Lipschitz continuous on $J \times B_{R}$ with respect to $u$, then the condition (4) is satisfied, but $f$ may not be necessarily uniformly continuous on $J \times B_{R}$.

Motivated by the above mentioned aspects, in this paper we studied the existence of mild solutions and positive mild solutions for the NPFEE (1) by utilizing a new fixed point theorem with respect to convex-power condensing operator due to Sun and Zhang [37] (see Lemma 8). Furthermore, we deleted the assumption that $f$ is uniformly continuous by using a new estimation technique of the measure of noncompactness (see Lemma 7).

\section{Preliminaries}

In this section, we introduce some notations, definitions and preliminary facts which are used throughout this paper.

Let $E$ be a real Banach space with the norm $\|\cdot\|$. We denote by $C(J, E)$ the Banach space of all continuous $E$ value functions on interval $J$ with the supnorm $\|u\|_{C}=$ $\sup _{t \in J}\|u(t)\|$, and by $L^{1}(J, E)$ the Banach space of all $E$-value Bochner integrable functions defined on $J$ with the norm $\|u\|_{1}=\int_{0}^{1}\|u(t)\| d t$

Definition 1 (see [3]). The fractional integral of order $q>0$ with the lower limit zero for a function $u \in L^{1}(J, E)$ is defined as

$$
I_{t}^{q} u(t)=\frac{1}{\Gamma(q)} \int_{0}^{t}(t-s)^{q-1} u(s) d s, \quad t>0
$$

where $\Gamma(\cdot)$ is the Euler gamma function. 
Definition 2 (see [3]). The Caputo fractional derivative of order $q>0$ with the lower limit zero for a function $u$ is defined as

$$
\begin{array}{r}
{ }^{C} D_{t}^{q} u(t)=\frac{1}{\Gamma(n-q)} \int_{0}^{t}(t-s)^{n-q-1} u^{(n)}(s) d s, \\
t>0, \quad 0 \leq n-1<q<n,
\end{array}
$$

where the function $u(t)$ has absolutely continuous derivatives up to order $n-1$.

If $u$ is an abstract function with values in $E$, then the integrals which appear in Definitions 1 and 2 are taken in Bochner's sense.

For $u \in E$, define two operators $\mathscr{T}_{q}(t)(t \geq 0)$ and $\mathcal{S}_{q}(t)(t \geq 0)$ by

$$
\begin{aligned}
& \mathscr{T}_{q}(t) u=\int_{0}^{\infty} h_{q}(s) T\left(t^{q} s\right) u d s \\
& \mathcal{S}_{q}(t) u=q \int_{0}^{\infty} s h_{q}(s) T\left(t^{q} s\right) u d s
\end{aligned}
$$

where

$$
h_{q}(s)=\frac{1}{\pi q} \sum_{n=1}^{\infty}(-s)^{n-1} \frac{\Gamma(n q+1)}{n !} \sin (n \pi q), \quad s \in(0, \infty)
$$

is the function of Wright type defined on $(0, \infty)$ which satisfies

$$
\begin{gathered}
h_{q}(s) \geq 0, \quad s \in(0, \infty), \quad \int_{0}^{\infty} h_{q}(s) d s=1, \\
\int_{0}^{\infty} s^{v} h_{q}(s) d s=\frac{\Gamma(1+v)}{\Gamma(1+q v)}, \quad v \in[0,1] .
\end{gathered}
$$

Let $M=\sup _{t \in[0,+\infty)}\|T(t)\|_{\mathscr{L}(E)}$, where $\mathscr{L}(E)$ stands for the Banach space of all linear and bounded operators in $E$. The following lemma follows from the results in $[12,13,20]$.

Lemma 3. The operators $\mathscr{T}_{q}(t)(t \geq 0)$ and $\mathcal{S}_{q}(t)(t \geq 0)$ have the following properties.

(1) For any fixed $t \geq 0, \mathscr{T}_{q}(t)$ and $\mathcal{S}_{q}(t)$ are linear and bounded operators; that is, for any $u \in E$,

$$
\begin{gathered}
\left\|\mathscr{T}_{q}(t) u\right\| \leq M\|u\|, \\
\left\|\mathcal{S}_{q}(t) u\right\| \leq \frac{q M}{\Gamma(1+q)}\|u\|=\frac{M}{\Gamma(q)}\|u\| .
\end{gathered}
$$

(2) For every $u \in E, t \rightarrow \mathscr{T}_{q}(t) u$ and $t \rightarrow \mathcal{S}_{q}(t) u$ are continuous functions from $[0, \infty)$ into $E$.

(3) The operators $\mathscr{T}_{q}(t)(t \geq 0)$ and $\mathcal{S}_{q}(t)(t \geq 0)$ are strongly continuous, which means that, for all $u \in E$ and $0 \leq t^{\prime}<t^{\prime \prime} \leq a$, one has

$$
\begin{gathered}
\left\|\mathscr{T}_{q}\left(t^{\prime \prime}\right) u-\mathscr{T}_{q}\left(t^{\prime}\right) u\right\| \longrightarrow 0, \\
\left\|\mathcal{S}_{q}\left(t^{\prime \prime}\right) u-\mathcal{S}_{q}\left(t^{\prime}\right) u\right\| \longrightarrow 0 \text { as } t^{\prime \prime}-t^{\prime} \longrightarrow 0 .
\end{gathered}
$$

Definition 4. A function $u \in C(J, E)$ is said to be a mild solution of the NPFEE (1) if it satisfies

$$
\begin{aligned}
u(t)= & \mathscr{T}_{q}(t) g(u)+\int_{0}^{t}(t-s)^{q-1} \\
& \times \mathcal{S}_{q}(t-s) f(s, u(s), G u(s), S u(s)) d s .
\end{aligned}
$$

Next, we recall some properties of the measure of noncompactness that will be used in the proof of our main results. Since no confusion may occur, we denote by $\alpha(\cdot)$ the Kuratowski measure of noncompactness on both the bounded sets of $E$ and $C(J, E)$. For the details of the definition and properties of the measure of noncompactness, we refer to the monographs $[39,40]$. For any $D \subset C(J, E)$ and $t \in J$, set $D(t)=\{u(t) \mid u \in D\} \subset E$. If $D \subset C(J, E)$ is bounded, then $D(t)$ is bounded in $E$ and $\alpha(D(t)) \leq \alpha(D)$.

Lemma 5 (see [39]). Let $E$ be a Banach space; let $D \subset C(J, E)$ be bounded and equicontinuous. Then $\alpha(D(t))$ is continuous on $J$, and

$$
\alpha(D)=\max _{t \in J} \alpha(D(t))=\alpha(D(J))
$$

Lemma 6 (see [41]). Let $E$ be a Banach space; let $D=$ $\left\{u_{n}\right\} \subset C(J, E)$ be a bounded and countable set. Then $\alpha(D(t))$ is Lebesgue integral on $\mathrm{J}$, and

$$
\alpha\left(\left\{\int_{J} u_{n}(t) d t \mid n \in \mathbb{N}\right\}\right) \leq 2 \int_{J} \alpha(D(t)) d t
$$

Lemma 7 (see $[32,42]$ ). Let $E$ be a Banach space; let $D \subset E$ be bounded. Then there exists a countable set $D_{0} \subset D$, such that $\alpha(D) \leq 2 \alpha\left(D_{0}\right)$.

Lemma 8 (fixed point theorem with respect to convex-power condensing operator (see [37])). Let E be a Banach space; let $D \subset E$ be bounded, closed, and convex. Suppose $Q: D \rightarrow D$ is a continuous operator and $Q(D)$ is bounded. For any $S \subset D$ and $x_{0} \in D$, set

$$
\begin{gathered}
Q^{\left(1, x_{0}\right)}(S) \equiv Q(S), \\
Q^{\left(n, x_{0}\right)}(S)=Q\left(\overline{c o}\left\{Q^{\left(n-1, x_{0}\right)}(S), x_{0}\right\}\right), \quad n=2,3, \ldots
\end{gathered}
$$

If there exist $x_{0} \in D$ and positive integer $n_{0}$ such that for any bounded and nonprecompact subset $S \subset D$,

$$
\alpha\left(Q^{\left(n_{0}, x_{0}\right)}(S)\right)<\alpha(S),
$$

then $Q$ has at least one fixed point in D.

Lemma 9. For $\sigma \in(0,1]$ and $0<a \leq b$, one has

$$
\left|a^{\sigma}-b^{\sigma}\right| \leq(b-a)^{\sigma} \text {. }
$$

\section{Existence of Mild Solutions}

In this section, we discuss the existence of mild solutions for the NPFEE (1). We first make the following hypotheses. 
(H1) The function $f: J \times E \times E \times E \rightarrow E$ satisfies the Carathéodory type conditions; that is, $f(\cdot, u, G u, S u)$ is strongly measurable for all $u \in E$, and $f(t, \cdot, \cdot, \cdot)$ is continuous for a.e. $t \in J$.

(H2) For some $r>0$, there exist constants $q_{1} \in[0, q), \rho_{1}>$ 0 and functions $\varphi_{r} \in L^{1 / q_{1}}\left(J, \mathbb{R}^{+}\right)$such that for a.e. $t \in J$ and all $u \in E$ satisfying $\|u\| \leq r$,

$$
\begin{gathered}
\|f(t, u, G u, S u)\| \leq \varphi_{r}(t), \\
\liminf _{r \rightarrow+\infty} \frac{\left\|\varphi_{r}\right\|_{L^{1 / q_{1}}[0, a]}}{r}=\rho_{1}<+\infty .
\end{gathered}
$$

(H3) There exist positive constants $L_{i}(i=1,2,3)$ such that for any bounded and countable sets $D_{i} \subset E(i=$ $1,2,3)$ and a.e. $t \in J$ :

$$
\alpha\left(f\left(t, D_{1}, D_{2}, D_{3}\right)\right) \leq \sum_{i=1}^{3} L_{i} \alpha\left(D_{i}\right) .
$$

(H4) The nonlocal function $g: C(J, E) \rightarrow E$ is continuous and compact, and there exist a constant $\rho_{2}>0$ and a nondecreasing continuous function $\Phi: \mathbb{R}^{+} \rightarrow \mathbb{R}^{+}$ such that, for some $r>0$ and all $u \in \Omega_{r}=\{u \in$ $\left.C(J, E):\|u\|_{C} \leq r\right\}$,

$$
\begin{gathered}
\|g(u)\| \leq \Phi(r), \\
\liminf _{r \rightarrow+\infty} \frac{\Phi(r)}{r}=\rho_{2}<+\infty .
\end{gathered}
$$

Theorem 10. Let $E$ be a real Banach space; let $A: D(A) \subset$ $E \rightarrow E$ be a closed linear operator, and $-A$ generate an equicontinuous $C_{0}$-semigroup $T(t)(t \geq 0)$ of uniformly bounded operators in E. Assume that the hypotheses (H1), (H2), (H3) and (H4) are satisfied, then the NPFEE (1) have at least one mild solution in $C(J, E)$ provided that

$$
M \rho_{2}+\frac{M \rho_{1} a^{q-q_{1}}}{\Gamma(q)}\left(\frac{1-q_{1}}{q-q_{1}}\right)^{1-q_{1}}<1 .
$$

Proof. Consider the operator $Q: C(J, E) \rightarrow C(J, E)$ defined by

$$
\begin{aligned}
(Q u)(t)= & \mathscr{T}_{q}(t) g(u)+\int_{0}^{t}(t-s)^{q-1} \\
& \times \mathcal{S}_{q}(t-s) f(s, u(s), G u(s), S u(s)) d s, \quad t \in J .
\end{aligned}
$$

By direct calculation, we know that $Q$ is well defined. From Definition 4, it is easy to see that the mild solution of the NPFEE (1) is equivalent to the fixed point of the operator $Q$. In the following, we will prove $Q$ has a fixed point by applying the fixed point theorem with respect to convexpower condensing operator.

Firstly, we prove that there exists a positive constant $R$, such that $Q\left(\Omega_{R}\right) \subset \Omega_{R}$. If this is not true, then for each $r>0$, there would exist $u_{r} \in \Omega_{r}$ and $t_{r} \in J$ such that
$\left\|\left(Q u_{r}\right)\left(t_{r}\right)\right\|>r$. It follows from Lemma 3 (1), the hypotheses $(\mathrm{H} 2)$ and (H4) and Hölder inequality that

$$
\begin{aligned}
r< & \left\|\left(Q u_{r}\right)\left(t_{r}\right)\right\| \\
\leq & M\left\|g\left(u_{r}\right)\right\| \\
& +\frac{M}{\Gamma(q)} \int_{0}^{t_{r}}\left(t_{r}-s\right)^{q-1} \\
& \times\left\|f\left(s, u_{r}(s), G u_{r}(s), S u_{r}(s)\right)\right\| d s \\
\leq & M \Phi(r)+\frac{M}{\Gamma(q)}\left(\int_{0}^{t_{r}}\left(t_{r}-s\right)^{(q-1) /\left(1-q_{1}\right)} d s\right)^{1-q_{1}} \\
& \times\left\|\varphi_{r}\right\|_{L^{1 / q_{1}}\left[0, t_{r}\right]} \\
\leq & M \Phi(r)+\frac{M a^{q-q_{1}}}{\Gamma(q)}\left(\frac{1-q_{1}}{q-q_{1}}\right)^{1-q_{1}}\left\|\varphi_{r}\right\|_{L^{1 / q_{1}}[0, a]} .
\end{aligned}
$$

Dividing both sides of (25) by $r$ and taking the lower limit as $r \rightarrow+\infty$, we get

$$
M \rho_{2}+\frac{M \rho_{1} a^{q-q_{1}}}{\Gamma(q)}\left(\frac{1-q_{1}}{q-q_{1}}\right)^{1-q_{1}} \geq 1,
$$

which contradicts (23).

Secondly, we prove that $Q$ is continuous in $\Omega_{R}$. To this end, let $\left\{u_{n}\right\}_{n=1}^{\infty} \subset \Omega_{R}$ be a sequence such that $\lim _{n \rightarrow+\infty} u_{n}=u$ in $\Omega_{R}$. By the continuity of $g$ and the Carathéodory continuity of $f$, we have

$$
\begin{gathered}
\lim _{n \rightarrow+\infty} g\left(u_{n}\right)=g(u), \\
\lim _{n \rightarrow+\infty} f\left(s, u_{n}(s), G u_{n}(s), S u_{n}(s)\right) \\
=f(s, u(s), G u(s), S u(s)), \quad \text { a.e. } s \in J .
\end{gathered}
$$

From the hypothesis (H2), we get for each $t \in J$

$$
\begin{gathered}
(t-s)^{q-1} \| f\left(s, u_{n}(s), G u_{n}(s), S u_{n}(s)\right) \\
-f(s, u(s), G u(s), S u(s)) \| \\
\leq 2(t-s)^{q-1} \varphi_{R}(s)
\end{gathered}
$$

Using the fact that the function $s \rightarrow 2(t-s)^{q-1} \varphi_{R}(s)$ is Lebesgue integrable for $s \in[0, t], t \in J$, by (27) and (28) and the Lebesgue dominated convergence theorem, we get that

$$
\begin{gathered}
\left\|\left(Q u_{n}\right)(t)-(Q u)(t)\right\| \\
\leq M\left\|g\left(u_{n}\right)-g(u)\right\| \\
+\frac{M}{\Gamma(q)}
\end{gathered}
$$




$$
\begin{aligned}
\times \int_{0}^{t}(t-s)^{q-1} & \\
\times \| f\left(s, u_{n}(s), G u_{n}(s), S u_{n}(s)\right) & \\
\quad-f(s, u(s), G u(s), S u(s)) \| d s & \longrightarrow 0 \\
\text { as } n & \longrightarrow \infty
\end{aligned}
$$

Therefore, we know that

$$
\left\|\left(Q u_{n}\right)-(Q u)\right\|_{C} \longrightarrow 0 \quad \text { as } n \longrightarrow \infty
$$

which means that $Q$ is continuous.

Now, we demonstrate that the operator $Q: \Omega_{R} \rightarrow \Omega_{R}$ is equicontinuous. For any $u \in \Omega_{R}$ and $0 \leq t_{1}<t_{2} \leq a$, we get that

$$
\begin{aligned}
& (Q u)\left(t_{2}\right)-(Q u)\left(t_{1}\right) \\
& =\mathscr{T}_{q}\left(t_{2}\right) g(u)-\mathscr{T}_{q}\left(t_{1}\right) g(u) \\
& \quad+\int_{t_{1}}^{t_{2}}\left(t_{2}-s\right)^{q-1} \mathcal{S}_{q}\left(t_{2}-s\right) \\
& \quad \times f(s, u(s), G u(s), S u(s)) d s \\
& +\int_{0}^{t_{1}}\left(\left(t_{2}-s\right)^{q-1}-\left(t_{1}-s\right)^{q-1}\right) \\
& \quad \times \mathcal{S}_{q}\left(t_{2}-s\right) f(s, u(s), G u(s), S u(s)) d s \\
& +\int_{0}^{t_{1}}\left(t_{1}-s\right)^{q-1}\left(\mathcal{S}_{q}\left(t_{2}-s\right)-\mathcal{S}_{q}\left(t_{1}-s\right)\right) \\
& \quad \times f(s, u(s), G u(s), S u(s)) d s \\
& =I_{1}+I_{2}+I_{3}+I_{4},
\end{aligned}
$$

where

$$
\begin{aligned}
& I_{1}=\mathscr{T}_{q}\left(t_{2}\right) g(u)-\mathscr{T}_{q}\left(t_{1}\right) g(u), \\
& \begin{aligned}
I_{2}=\int_{t_{1}}^{t_{2}}\left(t_{2}-s\right)^{q-1} \mathcal{S}_{q}\left(t_{2}-s\right) \\
\quad \times f(s, u(s), G u(s), S u(s)) d s, \\
I_{3}=\int_{0}^{t_{1}}\left(\left(t_{2}-s\right)^{q-1}-\left(t_{1}-s\right)^{q-1}\right) \\
\quad \times \mathcal{S}_{q}\left(t_{2}-s\right) f(s, u(s), G u(s), S u(s)) d s, \\
I_{4}=\int_{0}^{t_{1}}\left(t_{1}-s\right)^{q-1}\left(\mathcal{S}_{q}\left(t_{2}-s\right)-\mathcal{S}_{q}\left(t_{1}-s\right)\right) \\
\quad \times f(s, u(s), G u(s), S u(s)) d s .
\end{aligned}
\end{aligned}
$$

It is obvious that

$$
\left\|(Q u)\left(t_{2}\right)-(Q u)\left(t_{1}\right)\right\| \leq \sum_{i=1}^{4}\left\|I_{i}\right\| .
$$

Therefore, we only need to check $\left\|I_{i}\right\|$ tend to 0 independently of $u \in \Omega_{R}$ when $t_{2}-t_{1} \rightarrow 0, i=1,2, \ldots, 4$.

For $I_{1}$, by Lemma 3(3), $\left\|I_{1}\right\| \rightarrow 0$ as $t_{2}-t_{1} \rightarrow 0$.

For $I_{2}$, by the hypothesis (H2), Lemma 3(1), and Hölder inequality, we have

$$
\begin{aligned}
\left\|I_{2}\right\| \leq & \frac{M}{\Gamma(q)} \int_{t_{1}}^{t_{2}}\left(t_{2}-s\right)^{q-1} \varphi_{R}(s) d s \\
\leq & \frac{M}{\Gamma(q)}\left(\int_{t_{1}}^{t_{2}}\left(t_{2}-s\right)^{(q-1) /\left(1-q_{1}\right)} d s\right)^{1-q_{1}} \\
& \times\left\|\varphi_{R}\right\|_{L^{1 / q_{1}}\left[t_{1}, t_{2}\right]} \\
\leq & \frac{M\left\|\varphi_{R}\right\|_{L^{1 / q_{1}}[0, a]}}{\Gamma(q)}\left(\frac{1-q_{1}}{q-q_{1}}\right)^{1-q_{1}}\left(t_{2}-t_{1}\right)^{q-q_{1}} \longrightarrow 0 \\
& \text { as } t_{2}-t_{1} \longrightarrow 0 .
\end{aligned}
$$

For $I_{3}$, by the hypothesis (H2), Lemmas 3(1), and 9 and Hölder inequality, we get that

$$
\begin{aligned}
& \left\|I_{3}\right\| \leq \frac{M}{\Gamma(q)} \int_{0}^{t_{1}}\left(\left(t_{1}-s\right)^{q-1}-\left(t_{2}-s\right)^{q-1}\right) \varphi_{R}(s) d s \\
& \leq \frac{M}{\Gamma(q)}\left(\int_{0}^{t_{1}}\left(\left(t_{1}-s\right)^{q-1}-\left(t_{2}-s\right)^{q-1}\right)^{1 /\left(1-q_{1}\right)} d s\right)^{1-q_{1}} \\
& \times\left\|\varphi_{R}\right\|_{L^{1 / q_{1}}\left[0, t_{1}\right]} \\
& \leq \frac{M}{\Gamma(q)}\left(\int _ { 0 } ^ { t _ { 1 } } \left(\left(t_{1}-s\right)^{(q-1) /\left(1-q_{1}\right)}\right.\right. \\
& \left.\left.-\left(t_{2}-s\right)^{(q-1) /\left(1-q_{1}\right)}\right) d s\right)^{1-q_{1}} \\
& \times\left\|\varphi_{R}\right\|_{L^{1 / q_{1}}[0, a]} \\
& \leq \frac{M\left\|\varphi_{R}\right\|_{L^{1 / q_{1}[0, a]}}}{\Gamma(q)}\left(\frac{1-q_{1}}{q-q_{1}}\right)^{1-q_{1}} \\
& \times\left(t_{1}^{\left(q-q_{1}\right) /\left(1-q_{1}\right)}-t_{2}^{\left(q-q_{1}\right) /\left(1-q_{1}\right)}\right. \\
& \left.+\left(t_{2}-t_{1}\right)^{\left(q-q_{1}\right) /\left(1-q_{1}\right)}\right)^{1-q_{1}} \\
& \leq \frac{M 2^{1-q_{1}}\left\|\varphi_{R}\right\|_{L^{1 / q_{1}}[0, a]}}{\Gamma(q)}\left(\frac{1-q_{1}}{q-q_{1}}\right)^{1-q_{1}}\left(t_{2}-t_{1}\right)^{q-q_{1}} \\
& \longrightarrow 0 \quad \text { as } t_{2}-t_{1} \longrightarrow 0 \text {. }
\end{aligned}
$$


For $t_{1}=0,0<t_{2} \leq a$, it is easy to see that $\left\|I_{4}\right\|=0$. For $t_{1}>0$ and $\epsilon>0$ small enough, by the hypothesis (H2), Lemma 3 and the equicontinuity of $T(t)$, we know that

$$
\begin{aligned}
& \left\|I_{4}\right\| \leq \int_{0}^{t_{1}-\epsilon}\left(t_{1}-s\right)^{q-1} \\
& \times\left\|\mathcal{S}_{q}\left(t_{2}-s\right)-\mathcal{S}_{q}\left(t_{1}-s\right)\right\| \varphi_{R}(s) d s \\
& +\int_{t_{1}-\epsilon}^{t_{1}}\left(t_{1}-s\right)^{q-1} \\
& \times\left\|\mathcal{S}_{q}\left(t_{2}-s\right)-\mathcal{S}_{q}\left(t_{1}-s\right)\right\| \varphi_{R}(s) d s \\
& \leq \sup _{s \in\left[0, t_{1}-\epsilon\right]}\left\|\mathcal{S}_{q}\left(t_{2}-s\right)-\mathcal{S}_{q}\left(t_{1}-s\right)\right\| \\
& \times \int_{0}^{t_{1}-\epsilon}\left(t_{1}-s\right)^{q-1} \varphi_{R}(s) d s \\
& +\frac{2 M}{\Gamma(q)} \int_{t_{1}-\epsilon}^{t_{1}}\left(t_{1}-s\right)^{q-1} \varphi_{R}(s) d s \\
& \leq \sup _{s \in\left[0, t_{1}-\epsilon\right]}\left\|\mathcal{S}_{q}\left(t_{2}-s\right)-\mathcal{S}_{q}\left(t_{1}-s\right)\right\| \\
& \cdot\left\|\varphi_{R}\right\|_{L^{1 / q_{1}[0, a]}}\left(\frac{1-q_{1}}{q-q_{1}}\right)^{1-q_{1}} \\
& \cdot\left(t_{1}^{\left(q-q_{1}\right) /\left(1-q_{1}\right)}-\epsilon^{\left(q-q_{1}\right) /\left(1-q_{1}\right)}\right)^{1-q_{1}} \\
& +\frac{2 M\left\|\varphi_{R}\right\|_{L^{1 / q_{1}}[0, a]}}{\Gamma(q)}\left(\frac{1-q_{1}}{q-q_{1}}\right)^{1-q_{1}} \epsilon^{q-q_{1}} \longrightarrow 0 \\
& \text { as } t_{2}-t_{1} \longrightarrow 0 \text {. }
\end{aligned}
$$

As a result, $\left\|(Q u)\left(t_{2}\right)-(Q u)\left(t_{1}\right)\right\|$ tends to zero independently of $u \in \Omega_{R}$ as $t_{2}-t_{1} \rightarrow 0$, which means that $Q: \Omega_{R} \rightarrow \Omega_{R}$ is equicontinuous.

Let $F=\overline{\mathrm{co}} Q\left(\Omega_{R}\right)$, where $\overline{\mathrm{co}}$ means the closure of convex hull. Then it is easy to verify that $Q$ maps $F$ into itself and $F \subset C(J, E)$ is equicontinuous. Now, we are in the position to prove that $Q: F \rightarrow F$ is a convex-power condensing operator. Take $u_{0} \in F$; we will prove that there exists a positive integer $n_{0}$ such that for any bounded and nonprecompact subset $D \subset F$

$$
\alpha\left(Q^{\left(n_{0}, u_{0}\right)}(D)\right)<\alpha(D)
$$

For any $D \subset F$ and $u_{0} \in F$, by (17) and the equicontinuity of $F$, we get that $Q^{\left(n, u_{0}\right)}(D) \subset \Omega_{R}$ is also equicontinuous. Therefore, we know from Lemma 5 that

$$
\alpha\left(Q^{\left(n, u_{0}\right)}(D)\right)=\max _{t \in J} \alpha\left(Q^{\left(n, u_{0}\right)}(D)(t)\right), \quad n=1,2, \ldots
$$

By Lemma 7, there exists a countable set $D_{1}=\left\{u_{n}^{1}\right\} \subset D$, such that

$$
\alpha(Q(D)(t)) \leq 2 \alpha\left(Q\left(D_{1}\right)(t)\right) .
$$

Thanks to the fact that

$$
\int_{0}^{a} u(s) d s \in a \overline{\mathrm{co}}\{u(s) \mid s \in J\}, \quad u \in C(J, E)
$$

we have

$$
\begin{gathered}
\alpha\left(\left\{\int_{0}^{t} K(t, s) u(s) d s \mid u \in D, t \in J\right\}\right) \\
\leq a K_{0} \alpha(\{u(t) \mid u \in D, t \in J\}), \\
\alpha\left(\left\{\int_{0}^{a} H(t, s) u(s) d s \mid u \in D, t \in J\right\}\right) \\
\leq a H_{0} \alpha(\{u(t) \mid u \in D, t \in J\}),
\end{gathered}
$$

where $K_{0}=\max _{(t, s) \in \Delta}|K(t, s)|, H_{0}=\max _{(t, s) \in \Delta_{0}}|H(t, s)|$. Therefore, by (24), (39), and (41), Lemma 6 and the hypotheses (H3) and (H4), we get that

$$
\begin{aligned}
& \alpha\left(Q^{\left(1, u_{0}\right)}(D)(t)\right) \\
& =\alpha(Q(D)(t)) \leq 2 \alpha\left(Q\left(D_{1}\right)(t)\right) \\
& \leq 2 \alpha\left(\mathscr{T}_{q}(t) g\left(u_{n}^{1}\right)\right. \\
& \quad+\int_{0}^{t}(t-s)^{q-1} \mathcal{S}_{q}(t-s) \\
& \left.\quad \times f\left(s, u_{n}^{1}(s), G u_{n}^{1}(s), S u_{n}^{1}(s)\right) d s\right) \\
& \leq \frac{4 M}{\Gamma(q)} \int_{0}^{t}(t-s)^{q-1} \\
& \times \alpha\left(f\left(s, u_{n}^{1}(s), G u_{n}^{1}(s), S u_{n}^{1}(s)\right)\right) d s \\
& \leq \frac{4 M\left(L_{1}+a K_{0} L_{2}+a H_{0} L_{3}\right) t^{q}}{\Gamma(q)} \int_{0}^{t}(t-s)^{q-1} \\
& \quad \times\left[L_{1} \alpha\left(D_{1}(s)\right)+L_{2} \alpha\left(\left(G D_{1}\right)(s)\right)\right. \\
& \quad+\frac{4 M}{\Gamma(q)} \int_{0}^{t}(t-s)^{q-1} \\
& \times\left(L_{1}+a K_{0} L_{2}+a H_{0} L_{3}\right) \alpha\left(D_{1}(s)\right) d s \\
& \quad(s))] d s
\end{aligned}
$$

Again by Lemma 7, there exists a countable set $D_{2}=$ $\left\{u_{n}^{2}\right\} \subset \overline{\operatorname{co}}\left\{Q^{\left(1, u_{0}\right)}(D), u_{0}\right\}$, such that

$$
\alpha\left(Q\left(\overline{\operatorname{co}}\left\{Q^{\left(1, u_{0}\right)}(D), u_{0}\right\}\right)(t)\right) \leq 2 \alpha\left(Q\left(D_{2}\right)(t)\right)
$$


Therefore, by (24), (41), and (43), Lemma 6, and the hypotheses (H3) and (H4), we have that

$$
\begin{aligned}
& \alpha\left(Q^{\left(2, u_{0}\right)}(D)(t)\right) \\
& =\alpha\left(Q\left(\overline{\operatorname{co}}\left\{Q^{\left(1, u_{0}\right)}(D), u_{0}\right\}\right)(t)\right) \\
& \leq 2 \alpha\left(Q\left(D_{2}\right)(t)\right) \\
& \leq 2 \alpha\left(\int_{0}^{t}(t-s)^{q-1} \delta_{q}(t-s)\right. \\
& \left.\times f\left(s, u_{n}^{2}(s), G u_{n}^{2}(s), S u_{n}^{2}(s)\right) d s\right) \\
& \leq \frac{4 M}{\Gamma(q)} \int_{0}^{t}(t-s)^{q-1} \\
& \times \alpha\left(f\left(s, u_{n}^{2}(s), G u_{n}^{2}(s), S u_{n}^{2}(s)\right)\right) d s \\
& \leq \frac{4 M}{\Gamma(q)} \int_{0}^{t}(t-s)^{q-1} \\
& \times\left(L_{1}+a K_{0} L_{2}+a H_{0} L_{3}\right) \alpha\left(D_{2}(s)\right) d s \\
& \leq \frac{4 M\left(L_{1}+a K_{0} L_{2}+a H_{0} L_{3}\right)}{\Gamma(q)} \\
& \times \int_{0}^{t}(t-s)^{q-1} \cdot \alpha\left(\overline{c o}\left\{Q^{\left(1, u_{0}\right)}(D), u_{0}\right\}(s)\right) d s \\
& \leq \frac{\left[4 M\left(L_{1}+a K_{0} L_{2}+a H_{0} L_{3}\right)\right]^{2}}{\Gamma(q) \Gamma(1+q)} \\
& \times \int_{0}^{t}(t-s)^{q-1} s^{q} \alpha(D) d s \\
& =\frac{\left[4 M\left(L_{1}+a K_{0} L_{2}+a H_{0} L_{3}\right) t^{q}\right]^{2}}{\Gamma(1+2 q) \mathbf{B}(1+q, q)} \\
& \times \int_{0}^{1}(1-s)^{q-1} s^{q} d s \alpha(D) \\
& =\frac{\left[4 M\left(L_{1}+a K_{0} L_{2}+a H_{0} L_{3}\right) t^{q}\right]^{2}}{\Gamma(1+2 q)} \alpha(D) \text {, }
\end{aligned}
$$

where $\mathbf{B}(p, q)=\int_{0}^{1} s^{p-1}(1-s)^{q-1} d s$ is the Beta function. Suppose that

$$
\begin{aligned}
& \alpha\left(Q^{\left(k, u_{0}\right)}(D)(t)\right) \\
& \quad \leq \frac{\left[4 M\left(L_{1}+a K_{0} L_{2}+a H_{0} L_{3}\right) t^{q}\right]^{k}}{\Gamma(1+k q)} \alpha(D), \quad \forall t \in J .
\end{aligned}
$$

Then by Lemma 7, there exists a countable set $D_{k+1}=$ $\left\{u_{n}^{k+1}\right\} \subset \overline{\operatorname{co}}\left\{Q^{\left(k, u_{0}\right)}(D), u_{0}\right\}$, such that

$$
\alpha\left(Q\left(\overline{\operatorname{co}}\left\{Q^{\left(k, u_{0}\right)}(D), u_{0}\right\}\right)(t)\right) \leq 2 \alpha\left(Q\left(D_{k+1}\right)(t)\right) .
$$

From (24), (41), and (46), using Lemma 6, and the hypotheses $(\mathrm{H} 3)$ and $(\mathrm{H} 4)$, we get that

$$
\begin{aligned}
& \alpha\left(Q^{\left(k+1, u_{0}\right)}(D)(t)\right) \\
& =\alpha\left(Q\left(\overline{\operatorname{co}}\left\{Q^{\left(k, u_{0}\right)}(D), u_{0}\right\}\right)(t)\right) \\
& \leq 2 \alpha\left(Q\left(D_{k+1}\right)(t)\right) \\
& \leq 2 \alpha\left(\int_{0}^{t}(t-s)^{q-1} \mathcal{S}_{q}(t-s)\right. \\
& \left.\times f\left(s, u_{n}^{k+1}(s), G u_{n}^{k+1}(s), S u_{n}^{k+1}(s)\right) d s\right) \\
& \leq \frac{4 M}{\Gamma(q)} \int_{0}^{t}(t-s)^{q-1} \\
& \times\left(L_{1}+a K_{0} L_{2}+a H_{0} L_{3}\right) \alpha\left(D_{k+1}(s)\right) d s \\
& \leq \frac{4 M\left(L_{1}+a K_{0} L_{2}+a H_{0} L_{3}\right)}{\Gamma(q)} \\
& \times \int_{0}^{t}(t-s)^{q-1} \alpha\left(\overline{\operatorname{co}}\left\{Q^{\left(k, u_{0}\right)}(D), u_{0}\right\}(s)\right) d s \\
& \leq \frac{\left[4 M\left(L_{1}+a K_{0} L_{2}+a H_{0} L_{3}\right)\right]^{k+1}}{\Gamma(q) \Gamma(1+k q)} \\
& \times \int_{0}^{t}(t-s)^{q-1} s^{k q} \alpha(D) d s \\
& =\frac{\left[4 M\left(L_{1}+a K_{0} L_{2}+a H_{0} L_{3}\right) t^{q}\right]^{k+1}}{\Gamma(1+(k+1) q) \mathbf{B}(1+k q, q)} \\
& \times \int_{0}^{1}(1-s)^{q-1} s^{k q} d s \alpha(D) \\
& =\frac{\left[4 M\left(L_{1}+a K_{0} L_{2}+a H_{0} L_{3}\right) t^{q}\right]^{k+1}}{\Gamma(1+(k+1) q)} \alpha(D) \text {. }
\end{aligned}
$$

Hence, by the method of mathematical induction, for any positive integer $n$ and $t \in J$, we have

$$
\alpha\left(Q^{\left(n, u_{0}\right)}(D)(t)\right) \leq \frac{\left[4 M\left(L_{1}+a K_{0} L_{2}+a H_{0} L_{3}\right) t^{q}\right]^{n}}{\Gamma(1+n q)} \alpha(D) .
$$

Consequently, from (38) and (48), we have

$$
\begin{aligned}
\alpha\left(Q^{\left(n, u_{0}\right)}(D)\right) & =\max _{t \in J} \alpha\left(Q^{\left(n, u_{0}\right)}(D)(t)\right) \\
& \leq \frac{\left[4 M\left(L_{1}+a K_{0} L_{2}+a H_{0} L_{3}\right) a^{q}\right]^{n}}{\Gamma(1+n q)} \alpha(D) .
\end{aligned}
$$

By the well-known Stirling's formula, we know that

$$
\Gamma(1+n q)=\sqrt{2 \pi n q}\left(\frac{n q}{e}\right)^{n q} e^{\nu / 12 n q}, \quad 0<v<1 .
$$


Thus,

$$
\begin{aligned}
\frac{\left[4 M\left(L_{1}+a K_{0} L_{2}+a H_{0} L_{3}\right) a^{q}\right]^{n}}{\Gamma(1+n q)} \\
=\frac{\left[4 M\left(L_{1}+a K_{0} L_{2}+a H_{0} L_{3}\right) a^{q}\right]^{n}}{\sqrt{2 \pi n q}(n q / e)^{n q} e^{v / 12 n q}} \\
\quad \longrightarrow 0 \text { as } n \longrightarrow \infty .
\end{aligned}
$$

Therefore, there exists a large enough positive integer $n_{0}$ such that

$$
\frac{\left[4 M\left(L_{1}+a K_{0} L_{2}+a H_{0} L_{3}\right) a^{q}\right]^{n_{0}}}{\Gamma\left(1+n_{0} q\right)}<1 .
$$

Hence, we get that

$$
\alpha\left(Q^{\left(n_{0}, u_{0}\right)}(D)\right)<\alpha(D)
$$

Thus, $Q: F \rightarrow F$ is a convex-power condensing operator. It follows from Lemma 8 that $Q$ has at least one fixed point $u \in F$, which is just a mild solution of the NPFEE (1). This completes the proof of Theorem 10 .

If we replace the hypotheses (H2) and (H4) by the following hypotheses:

$(\mathrm{H} 2)^{\prime}$ there exist a function $\varphi \in L^{1 / q_{1}}\left(J, \mathbb{R}^{+}\right), q_{1} \in[0, q)$ and a nondecreasing continuous function $\Psi: \mathbb{R}^{+} \rightarrow \mathbb{R}^{+}$ such that

$$
\|f(t, u, G u, S u)\| \leq \varphi(t) \Psi(\|u\|),
$$

for all $u \in E$ and a.e. $t \in J$;

$(\mathrm{H} 4)^{\prime}$ the nonlocal function $g: C(J, E) \rightarrow E$ is continuous and compact; there exist constants $0<b<1 / M$ and $c>0$ such that for all $u \in C(J, E),\|g(u)\| \leq b\|u\|_{C}+c$;

we have the following existence result.

Theorem 11. Let $E$ be a real Banach space; let $A: D(A) \subset$ $E \rightarrow E$ be a closed linear operator and $-A$ generate an equicontinuous $C_{0}$-semigroup $T(t)(t \geq 0)$ of uniformly bounded operators in E. Assume that the hypotheses (H1), $(\mathrm{H} 2)^{\prime},(\mathrm{H} 3)$, and $(\mathrm{H} 4)^{\prime}$ are satisfied, then the NPFEE (1) has at least one mild solution in $C(J, E)$ provided that there exists a constant $R$ with

$$
\begin{aligned}
(M c+ & \left.\frac{\Psi(R) M a^{q-q_{1}}}{\Gamma(q)}\left(\frac{1-q_{1}}{q-q_{1}}\right)^{1-q_{1}}\|\varphi\|_{L^{1 / q_{1}}[0, a]}\right) \\
& \times(1-M b)^{-1} \leq R .
\end{aligned}
$$

Proof. From the proof of Theorem 10, we know that the mild solution of the NPFEE (1) is equivalent to the fixed point of the operator $Q$ defined by (24). For any $u \in \Omega_{R}$, by (24), (55), and the hypotheses $(\mathrm{H} 2)^{\prime}$ and $(\mathrm{H} 4)^{\prime}$, we have

$$
\begin{aligned}
\|(Q u)(t)\| \leq & M\|g(u)\| \\
& +\frac{M}{\Gamma(q)} \int_{0}^{t}(t-s)^{q-1} \\
& \times\|f(s, u(s), G u(s), S u(s))\| d s \\
\leq & M\left(b\|u\|_{C}+c\right)+\frac{\Psi(R) M}{\Gamma(q)} \\
& \times\left(\int_{0}^{t}(t-s)^{(q-1) /\left(1-q_{1}\right)} d s\right)^{1-q_{1}}\|\varphi\|_{L^{1 / q_{1}}[0, t]} \\
\leq & M(b R+c)+\frac{\Psi(R) M a^{q-q_{1}}}{\Gamma(q)} \\
& \times\left(\frac{1-q_{1}}{q-q_{1}}\right)^{1-q_{1}}\|\varphi\|_{L^{1 / q_{1}}[0, a]} \\
\leq & R, \quad
\end{aligned}
$$

which implies $Q\left(\Omega_{R}\right) \subset \Omega_{R}$. By adopting completely similar method with the proof of Theorem 10, we can prove that the NPFEE (1) have at least one mild solution in $C(J, E)$. This completes the proof of Theorem 11.

Similar with Theorem 11, we have the following result.

Corollary 12. Let $E$ be a real Banach space; let $A: D(A) \subset$ $E \rightarrow E$ be a closed linear operator and $-A$ generate an equicontinuous $C_{0}$-semigroup $T(t)(t \geq 0)$ of uniformly bounded operators in E. Assume that the hypotheses (H1), $(\mathrm{H} 2)^{\prime},(\mathrm{H} 3)$ and $(\mathrm{H} 4)^{\prime}$ are satisfied, then the NPFEE (1) have at least one mild solution in $C(J, E)$ provided that

$$
\begin{aligned}
\left(\int_{0}^{a} \varphi^{1 / q_{1}}(s) d s\right)^{q_{1}} & <\liminf _{r \rightarrow+\infty} \frac{(r(1-M b)+M c) \Gamma(q)}{\Psi(r) M a^{q-q_{1}}} \\
& \times\left(\frac{q-q_{1}}{1-q_{1}}\right)^{1-q_{1}} .
\end{aligned}
$$

\section{Existence of Positive Mild Solutions}

In this section, we discuss the existence of positive mild solutions for the NPFEE (1). we first introduce some notations and definitions which will be used in this section.

Let $E$ be an ordered Banach space with the norm $\|$ - \| and let $P=\{u \in E \mid u \geq \theta\}$ be a positive cone in $E$ which defines a partial ordering in $E$ by $x \leq y$ if and only if $y-x \in P$, where $\theta$ denotes the zero element of $E$. $P$ is said to be normal if there exists a positive constant $N$ such that $\theta \leq x \leq y$ implies $\|x\| \leq N\|y\|$; the infimum of all $N$ with the property above is called the normal constant of $P$. For more definitions and details of the cone $P$, we refer to the monographs $[40,43]$. 
Definition 13. A $C_{0}$-semigroup $T(t)(t \geq 0)$ in $E$ is called to be positive, if order inequality $T(t) u \geq \theta$ holds for each $u \geq \theta$, $u \in E$ and $t \geq 0$.

For more details of the properties of positive $C_{0^{-}}$ semigroup, we refer to the monograph [44] and the paper [45].

Lemma 14. If $T(t)(t \geq 0)$ is a positive $C_{0}$-semigroup in $E$, then $\mathscr{T}_{q}(t)(t \geq 0)$ and $\mathcal{S}_{q}(t)(t \geq 0)$ are also positive operators.

Proof. Since the semigroup $T(t)(t \geq 0)$ and the function $h_{q}(s)$ defined by (10) are positive, by (9) we can easily conclude that the operators $\mathscr{T}_{q}(t)(t \geq 0)$ and $\mathcal{S}_{q}(t)(t \geq 0)$ are also positive. This completes the proof.

Here, we will obtain positive mild solutions under the following assumptions.

(A1) The function $f: J \times P \times P \times P \rightarrow P$ satisfies the Carathéodory type conditions.

(A2) There exist a constant $q_{1} \in[0, q)$ and a function $\varphi \in$ $L^{1 / q_{1}}\left(J, \mathbb{R}^{+}\right)$such that, for all $u \in P$ and a.e. $t \in J$, $f(t, u, G u, S u) \leq \varphi(t)$.

(A3) There exist positive constants $L_{i}(i=1,2,3)$ such that for any bounded and countable sets $D_{i} \subset C(J, P)(i=$ $1,2,3)$ and a.e. $t \in J$,

$$
\alpha\left(f\left(t, D_{1}(t), D_{2}(t), D_{3}(t)\right)\right) \leq \sum_{i=1}^{3} L_{i} \alpha\left(D_{i}(t)\right)
$$

(A4) The nonlocal function $g: C(J, P) \rightarrow P$ is continuous and compact, and there exist constants $0<\mathrm{b}<$ $1 /(\mathrm{NM})$ and $\mathrm{c}>0$ such that for all $u \in C(J, P)$, $g(u) \leq b u+c$, where $N$ is the normal constant of the positive cone $P$.

Theorem 15. Let $E$ be an ordered Banach space, $P$ be a normal positive cone with normal constant $N, A: D(A) \subset E \rightarrow$ $E$ be a closed linear operator, and $-A$ generate a positive and equicontinuous $C_{0}$-semigroup $T(t)(t \geq 0)$ of uniformly bounded operators in E. Assume that the assumptions (A1), (A2), (A3), and (A4) are satisfied, then the NPFEE (1) have at least one positive mild solution in $C(J, P)$.

Proof. From the proof of Theorem 10, we know that the positive mild solution of the NPFEE (1) is equivalent to the fixed point of operator $Q$ defined by $(24)$ in $C(J, P)$. We choose $R>0$ big enough such that

$$
R \geq \frac{N M}{1-N M b}\left[c+\frac{a^{q-q_{1}}}{\Gamma(q)}\left(\frac{1-q_{1}}{q-q_{1}}\right)^{1-q_{1}}\|\varphi\|_{L^{1 / q_{1}[0, a]}}\right]
$$

Then for any $u \in \Omega_{R}(P)=\left\{u \in C(J, P):\|u\|_{C} \leq R\right\}$, by (24) and the assumptions (A2) and (A4), we have that

$$
\begin{aligned}
\theta \leq & (Q u)(t) \\
\leq & \mathscr{T}_{q}(t)(b u+c) \\
& \quad+\int_{0}^{t}(t-s)^{q-1} \mathcal{S}_{q}(t-s) \varphi(s) d s .
\end{aligned}
$$

By the normality of the cone $P,(59),(60)$, the assumption (A4), and Hölder inequality, we get

$$
\begin{aligned}
\|(Q u)(t)\| \leq & N M\left(b\|u\|_{C}+c\right) \\
& +\frac{N M}{\Gamma(q)}\left(\int_{0}^{t}(t-s)^{(q-1) /\left(1-q_{1}\right)} d s\right)^{1-q_{1}} \\
& \times\|\varphi\|_{L^{1 / q_{1}}[0, t]} \\
\leq & N M(b R+c)+\frac{N M a^{q-q_{1}}}{\Gamma(q)}\left(\frac{1-q_{1}}{q-q_{1}}\right)^{1-q_{1}} \\
& \times\|\varphi\|_{L^{1 / q_{1}}[0, a]} \leq R .
\end{aligned}
$$

Thus, $Q: \Omega_{R}(P) \rightarrow \Omega_{R}(P)$. Let $F=\overline{\mathrm{co}} Q\left(\Omega_{R}(P)\right)$. Similar to the proof of Theorem 10, we can prove that $Q: F \rightarrow F$ is a convex-power condensing operator. It follows from Lemma 8 that $Q$ has at least one fixed point $u \in F$, which is just a positive mild solution of the NPFEE (1). This completes the proof of Theorem 15.

Theorem 16. Let $E$ be an ordered Banach space, $P$ be a normal positive cone with normal constant $N, A: D(A) \subset E \rightarrow$ $E$ be a closed linear operator and $-A$ generate a positive and equicontinuous $C_{0}$-semigroup $T(t)(t \geq 0)$ of uniformly bounded operators in E. Assume that the assumptions (A1), (A3) and the following assumptions:

$(\mathrm{A} 2)^{*}$ There exist nonnegative continuous functions $g_{1}(t)$, $g_{2}(t)$ and abstract continuous function $h: J \rightarrow P$ such that

$$
\begin{array}{r}
f(t, u, v, w) \leq g_{1}(t) u+g_{2}(t) v+h(t), \\
\text { a.e. } t \in J, \forall u, v, w \in P,
\end{array}
$$

$(\mathrm{A} 4)^{*}$ The nonlocal function $g: C(J, P) \rightarrow P$ is continuous and compact, and there exists a constant $c>0$ such that, for any $u \in C(J, P), g(u) \leq c$,

are satisfied, then the NPFEE (1) have at least one positive mild solution in $C(J, P)$.

Proof. From the proof of Theorem 10, we know that the positive mild solution of the NPFEE (1) is equivalent to the fixed point of operator $Q$ defined by $(24)$ in $C(J, P)$. Let

$$
\begin{aligned}
(B u)(t)=\int_{0}^{t}( & t-s)^{q-1} \mathcal{S}_{q}(t-s) \\
& \times\left[g_{1}(s) u(s)+g_{2}(s) G u(s)\right] d s, \quad t \in J .
\end{aligned}
$$


We can prove that $r(B)=0$, where $r(\cdot)$ denotes the spectral radius of bounded linear operator. In fact, for any $t \in J$, by (63), we get that

$\|(B u)(t)\|$

$$
\begin{aligned}
& \leq \frac{M}{\Gamma(q)} \int_{0}^{t}(t-s)^{q-1} \\
& \quad \times\left\|g_{1}(s) u(s)+g_{2}(s) \int_{0}^{s} K(s, \tau) u(\tau) d \tau\right\| d s \\
& \leq \frac{M G^{*}\left(1+a K_{0}\right)\|u\|_{C}}{\Gamma(q)} \int_{0}^{t}(t-s)^{q-1} d s \\
& =\frac{\beta t^{q}\|u\|_{C}}{\Gamma(1+q)},
\end{aligned}
$$

where $G^{*}=\max \left\{\max _{t \in J} g_{1}(t), \max _{t \in J} g_{2}(t)\right\}, \beta=M G^{*}(1+$ $\left.a K_{0}\right)$. Further,

$$
\begin{aligned}
\left\|\left(B^{2} u\right)(t)\right\| & \frac{M G^{*}}{\Gamma(q)} \int_{0}^{t}(t-s)^{q-1} \\
\quad \times\left\|(B u)(s)+\int_{0}^{s} K(s, \tau)(B u)(\tau) d \tau\right\| d s & \\
\leq & \frac{M G^{*} \beta\|u\|_{C}}{\Gamma(q) \Gamma(1+q)} \\
& \times \int_{0}^{t}(t-s)^{q-1}\left[s^{q}+\int_{0}^{s} K(s, \tau) \tau^{q} d \tau\right] d s \\
\leq & \frac{\beta^{2}\|u\|_{C}}{\Gamma(1+2 q) \mathbf{B}(1+q, q)} \\
\times \int_{0}^{t}(t-s)^{q-1} s^{q} d s & \frac{\beta^{2} t^{2 q}\|u\|_{C}}{\Gamma(1+2 q)} .
\end{aligned}
$$

By the method of mathematical induction, for any positive integer $n$ and $t \in J$, we obtain that

$$
\left\|\left(B^{n} u\right)(t)\right\| \leq \frac{\beta^{n} t^{n q}\|u\|_{C}}{\Gamma(1+n q)} .
$$

Therefore, we have

$$
\left\|B^{n}\right\|_{C} \leq \frac{\beta^{n} a^{n q}}{\Gamma(1+n q)} .
$$

Thus, combining (50) we get that

$$
r(B)=\lim _{n \rightarrow \infty}\left\|B^{n}\right\|_{C}^{1 / n} \leq \lim _{n \rightarrow \infty} \frac{\beta a^{q}}{\sqrt[2 n]{2 \pi n q}(n q / e)^{q} e^{v /\left(12 n^{2} q\right)}}=0 .
$$

Let $0<\gamma<1 / N$. From [46] we know that there exists an equivalent norm $\|\cdot\|^{*}$ in $E$ such that

$$
\|B\|^{*} \leq r(B)+\gamma=\gamma,
$$

where $\|B\|^{*}$ denotes the operator norm of $B$ with respect to the norm $\|\cdot\|^{*}$.

Let $M^{*}=\sup _{t \in[0,+\infty)}\|T(t)\|_{\mathscr{L}(E)}^{*}$ and $\|u\|_{C}^{*}=$ $\sup _{t \in J}\|u(t)\|^{*}$. Choose

$$
R^{*}=\frac{N M^{*}\left(\Gamma(1+q) c+a^{q}\|h\|_{C}^{*}\right)}{\Gamma(1+q)(1-N \gamma)} .
$$

For any $u \in \Omega_{R^{*}}^{*}(P)=\left\{u \in C(J, P):\|u\|_{C}^{*} \leq R^{*}\right\}$, by $(24)$ and the assumptions $(\mathrm{A} 2)^{*}$ and $(\mathrm{A} 4)^{*}$, we have that

$$
\begin{aligned}
\theta \leq & (Q u)(t) \\
\leq & \mathscr{T}_{q}(t) g(u) \\
& +\int_{0}^{t}(t-s)^{q-1} \mathcal{S}_{q}(t-s) \\
\quad & \times\left[g_{1}(s) u(s)+g_{2}(s) G u(s)+h(s)\right] d s \\
\leq & \mathscr{T}_{q}(t) c+B(u)(t) \\
& +\int_{0}^{t}(t-s)^{q-1} \mathcal{S}_{q}(t-s) h(s) d s .
\end{aligned}
$$

By the normality of the cone $P,(69),(70)$, and (71), we have

$$
\begin{aligned}
\|(Q u)(t)\|^{*} \leq N\left(\left\|\mathscr{T}_{q}(t) c\right\|^{*}+\|(B u)(t)\|^{*}\right. \\
\left.\quad+\left\|\int_{0}^{t}(t-s)^{q-1} \delta_{q}(t-s) h(s) d s\right\|^{*}\right) \\
\leq N\left(M^{*} c+\|B\|^{*}\|u\|_{C}^{*}+\frac{M^{*} a^{q}\|h\|_{C}^{*}}{\Gamma(1+q)}\right) \\
\leq N M^{*} c+N \gamma R^{*}+\frac{N M^{*} a^{q}\|h\|_{C}^{*}}{\Gamma(1+q)} \\
=R^{*} .
\end{aligned}
$$

Thus, we have proved that $Q: \Omega_{R^{*}}^{*}(P) \rightarrow \Omega_{R^{*}}^{*}(P)$. Let $F=\overline{\mathrm{CO}} Q\left(\Omega_{R^{*}}^{*}(P)\right)$. Similar to the proof of Theorem 10, we can prove that $Q: F \rightarrow F$ is a convex-power condensing operator. It follows from Lemma 8 that $Q$ has at least one fixed point $u \in F$, which is just a positive mild solution of the NPFEE (1). This completes the proof of Theorem 16.

Remark 17. Positive operator semigroups are widely appearing in heat conduction equations, neutron transport equations, reaction diffusion equations, and so on [47]. Therefore, using Theorems 15 and 16 to these partial differential equations are very convenient.

Remark 18. Analytic semigroup and differentiable semigroup are equicontinuous semigroup [48]. In the application of 
partial differential equations, such as parabolic equations and strongly damped wave equations, the corresponding solution semigroup is analytic semigroup. Therefore, the results obtained in this paper have broad applicability.

\section{Acknowledgments}

This research was supported by NNSF of China (11261053 and 11061031), NSF of Gansu Province (1208RJZA129), and Project of NWNU-LKQN-11-3.

\section{References}

[1] K. S. Miller and B. Ross, An Introduction to the Fractional Calculus and Differential Equations, Wiley, New York, NY, USA, 1993.

[2] I. Podlubny, Fractional Differential Equations, Academic Press, San Diego, Calif, USA, 1999.

[3] A. A. Kilbas, H. M. Srivastava, and J. J. Trujillo, Theory and Applications of Fractional Differential Equations, vol. 204 of North-Holland Mathematics Studies, Elsevier Science B.V., Amsterdam, The Netherlands, 2006.

[4] S. D. Eidelman and A. N. Kochubei, "Cauchy problem for fractional diffusion equations," Journal of Differential Equations, vol. 199, no. 2, pp. 211-255, 2004.

[5] V. Lakshmikantham and A. S. Vatsala, "Basic theory of fractional differential equations," Nonlinear Analysis: Theory, Methods \& Applications, vol. 69, no. 8, pp. 2677-2682, 2008.

[6] R. P. Agarwal, V. Lakshmikantham, and J. J. Nieto, "On the concept of solution for fractional differential equations with uncertainty," Nonlinear Analysis: Theory, Methods \& Applications, vol. 72, no. 6, pp. 2859-2862, 2010.

[7] M. A. Darwish and S. K. Ntouyas, "Boundary value problems for fractional functional differential equations of mixed type," Communications in Applied Analysis, vol. 13, no. 1, pp. 31-38, 2009.

[8] M. A. Darwish and S. K. Ntouyas, "Semilinear functional differential equations of fractional order with state-dependent delay," Electronic Journal of Differential Equations, vol. 2009, no. 38, pp. 1-10, 2009.

[9] M. A. Darwish and S. K. Ntouyas, "On initial and boundary value problems for fractional order mixed type functional differential inclusions," Computers \& Mathematics with Applications, vol. 59, no. 3, pp. 1253-1265, 2010.

[10] M. A. Darwish and S. K. Ntouyas, "On a quadratic fractional Hammerstein-Volterra integral equation with linear modification of the argument," Nonlinear Analysis: Theory, Methods \& Applications, vol. 74, no. 11, pp. 3510-3517, 2011.

[11] M. A. Darwish, J. Henderson, and D. O’Regan, "Existence and asymptotic stability of solutions of a perturbed fractional functional-integral equation with linear modification of the argument," Bulletin of the Korean Mathematical Society, vol. 48, no. 3, pp. 539-553, 2011.

[12] M. M. El-Borai, "Some probability densities and fundamental solutions of fractional evolution equations," Chaos, Solitons and Fractals, vol. 14, no. 3, pp. 433-440, 2002.

[13] M. M. El-Borai, "Semigroups and some nonlinear fractional differential equations," Applied Mathematics and Computation, vol. 149, no. 3, pp. 823-831, 2004.
[14] J. Wang, Y. Zhou, and M. Fečkan, "Abstract Cauchy problem for fractional differential equations," Nonlinear Dynamics, vol. 71, no. 4, pp. 685-700, 2013.

[15] J. Wang, Y. Zhou, and M. Medved', “On the solvability and optimal controls of fractional integrodifferential evolution systems with infinite delay," Journal of Optimization Theory and Applications, vol. 152, no. 1, pp. 31-50, 2012.

[16] J. Wang, Y. Zhou, and W. Wei, "Optimal feedback control for semilinear fractional evolution equations in Banach spaces," Systems \& Control Letters, vol. 61, no. 4, pp. 472-476, 2012.

[17] T. Diagana, G. M. Mophou, and G. M. N’Guérékata, “On the existence of mild solutions to some semilinear fractional integro-differential equations," Electronic Journal of Qualitative Theory of Differential Equations, vol. 2010, no. 58, pp. 1-17, 2010.

[18] R. Wang, T. Xiao, and J. Liang, "A note on the fractional Cauchy problems with nonlocal initial conditions," Applied Mathematics Letters, vol. 24, no. 8, pp. 1435-1442, 2011.

[19] F. Li, J. Liang, and H. K. Xu, "Existence of mild solutions for fractional integrodifferential equations of Sobolev type with nonlocal conditions," Journal of Mathematical Analysis and Applications, vol. 391, no. 2, pp. 510-525, 2012.

[20] Y. Zhou and F. Jiao, "Nonlocal Cauchy problem for fractional evolution equations," Nonlinear Analysis: Real World Applications, vol. 11, no. 5, pp. 4465-4475, 2010.

[21] J. Wang, Y. Zhou, W. Wei, and H. Xu, "Nonlocal problems for fractional integrodifferential equations via fractional operators and optimal controls," Computers \& Mathematics with Applications, vol. 62, no. 3, pp. 1427-1441, 2011.

[22] J. Wang, M. Fečkan, and Y. Zhou, "On the new concept of solutions and existence results for impulsive fractional evolution equations," Dynamics of Partial Differential Equations, vol. 8, no. 4, pp. 345-361, 2011.

[23] J. Wang, Z. Fan, and Y. Zhou, "Nonlocal controllability of semilinear dynamic systems with fractional derivative in Banach spaces," Journal of Optimization Theory and Applications, vol. 154, no. 1, pp. 292-302, 2012.

[24] Y. Chang, V. Kavitha, and M. Mallika Arjunan, "Existence and uniqueness of mild solutions to a semilinear integrodifferential equation of fractional order," Nonlinear Analysis: Theory, Methods \& Applications, vol. 71, no. 11, pp. 5551-5559, 2009.

[25] K. Balachandran and J. Y. Park, "Nonlocal Cauchy problem for abstract fractional semilinear evolution equations," Nonlinear Analysis: Theory, Methods \& Applications, vol. 71, no. 10, pp. 4471-4475, 2009.

[26] K. Balachandran and J. J. Trujillo, "The nonlocal Cauchy problem for nonlinear fractional integrodifferential equations in Banach spaces," Nonlinear Analysis: Theory, Methods \& Applications, vol. 72, no. 12, pp. 4587-4593, 2010.

[27] L. Byszewski and V. Lakshmikantham, "Theorem about the existence and uniqueness of a solution of a nonlocal abstract Cauchy problem in a Banach space," Applicable Analysis, vol. 40, no. 1, pp. 11-19, 1991.

[28] L. Byszewski, "Theorems about the existence and uniqueness of solutions of a semilinear evolution nonlocal Cauchy problem," Journal of Mathematical Analysis and Applications, vol. 162, no. 2, pp. 494-505, 1991.

[29] L. Byszewski, "Application of properties of the right-hand sides of evolution equations to an investigation of nonlocal evolution problems," Nonlinear Analysis: Theory, Methods \& Applications, vol. 33, no. 5, pp. 413-426, 1998. 
[30] H. Liu and J.-C. Chang, "Existence for a class of partial differential equations with nonlocal conditions," Nonlinear Analysis: Theory, Methods \& Applications, vol. 70, no. 9, pp. 3076-3083, 2009.

[31] J. Wang and W. Wei, "A class of nonlocal impulsive problems for integrodifferential equations in Banach spaces," Results in Mathematics, vol. 58, no. 3-4, pp. 379-397, 2010.

[32] P. Chen and Y. Li, "Monotone iterative technique for a class of semilinear evolution equations with nonlocal conditions," Results in Mathematics, 2012.

[33] V. Lakshmikantham and S. Leela, Nonlinear Differential Equations in Abstract Spaces, Pergamon Press, New York, NY, USA, 1981.

[34] D. Guo, "Solutions of nonlinear integro-differential equations of mixed type in Banach spaces," Journal of Applied Mathematics and Simulation, vol. 2, no. 1, pp. 1-11, 1989.

[35] L. Liu, C. Wu, and F. Guo, "Existence theorems of global solutions of initial value problems for nonlinear integro-differential equations of mixed type in Banach spaces and applications," Computers \& Mathematics with Applications, vol. 47, no. 1, pp. 13-22, 2004.

[36] L. Liu, F. Guo, C. Wu, and Y. Wu, "Existence theorems of global solutions for nonlinear Volterra type integral equations in Banach spaces," Journal of Mathematical Analysis and Applications, vol. 309, no. 2, pp. 638-649, 2005.

[37] J. X. Sun and X. Y. Zhang, "The fixed point theorem of convex-power condensing operator and applications to abstract semilinear evolution equations," Acta Mathematica Sinica, vol. 48, no. 3, pp. 439-446, 2005 (Chinese).

[38] H. B. Shi, W. T. Li, and H. R. Sun, "Existence of mild solutions for abstract mixed type semilinear evolution equations," Turkish Journal of Mathematics, vol. 35, no. 3, pp. 457-472, 2011.

[39] J. Banaś and K. Goebel, Measures of Noncompactness in Banach Spaces, vol. 60 of Lecture Notes in Pure and Applied Mathematics, Marcel Dekker, New York, NY, USA, 1980.

[40] K. Deimling, Nonlinear Functional Analysis, Springer, New York, NY, USA, 1985.

[41] H. P. Heinz, "On the behaviour of measures of noncompactness with respect to differentiation and integration of vector-valued functions," Nonlinear Analysis: Theory, Methods \& Applications, vol. 7, no. 12, pp. 1351-1371, 1983.

[42] Y. X. Li, "Existence of solutions to initial value problems for abstract semilinear evolution equations," Acta Mathematica Sinica, vol. 48, no. 6, pp. 1089-1094, 2005 (Chinese).

[43] D. J. Guo and V. Lakshmikantham, Nonlinear Problems in Abstract Cones, Academic Press, Orlando, Fla, USA, 1988.

[44] J. Banasiak and L. Arlotti, Perturbations of Positive Semigroups with Applications, Springer, London, UK, 2006.

[45] Y. X. Li, "The positive solutions of abstract semilinear evolution equations and their applications," Acta Mathematica Sinica, vol. 39, no. 5, pp. 666-672, 1996 (Chinese).

[46] I. Vasile, Fixed Point Theory, D. Reidel Publishing, Dordrecht, The Netherlands, 1981.

[47] R. Nagel, W. Arendt, A. Grabosch et al., One Parameter Semigroups of Positive Operators, vol. 1184 of Lecture Notes in Mathematics, Springer, Berlin, Germany, 1986.

[48] A. Pazy, Semigroups of Linear Operators and Applications to Partial Differential Equations, Springer, Berlin, Germany, 1983. 


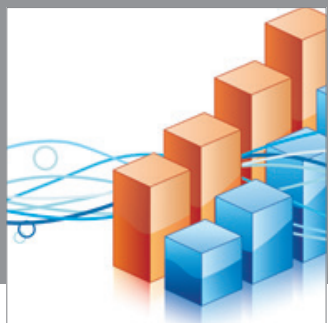

Advances in

Operations Research

mansans

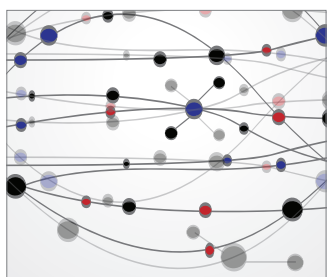

The Scientific World Journal
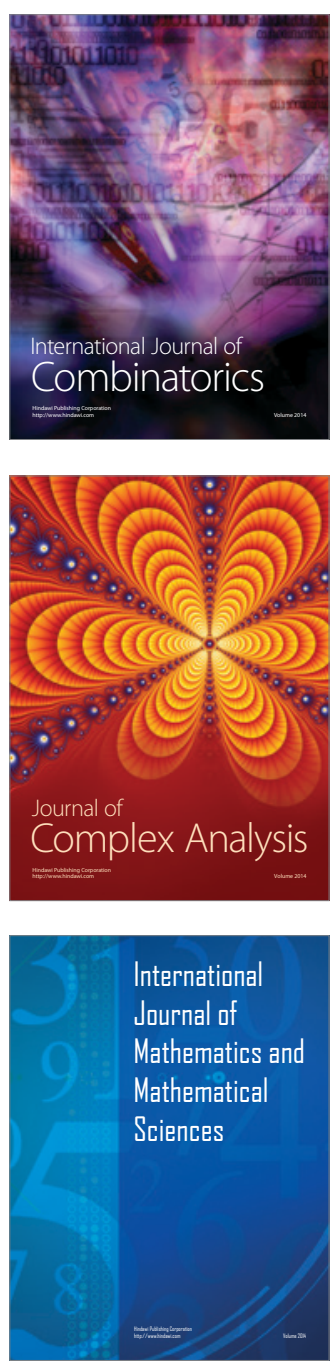
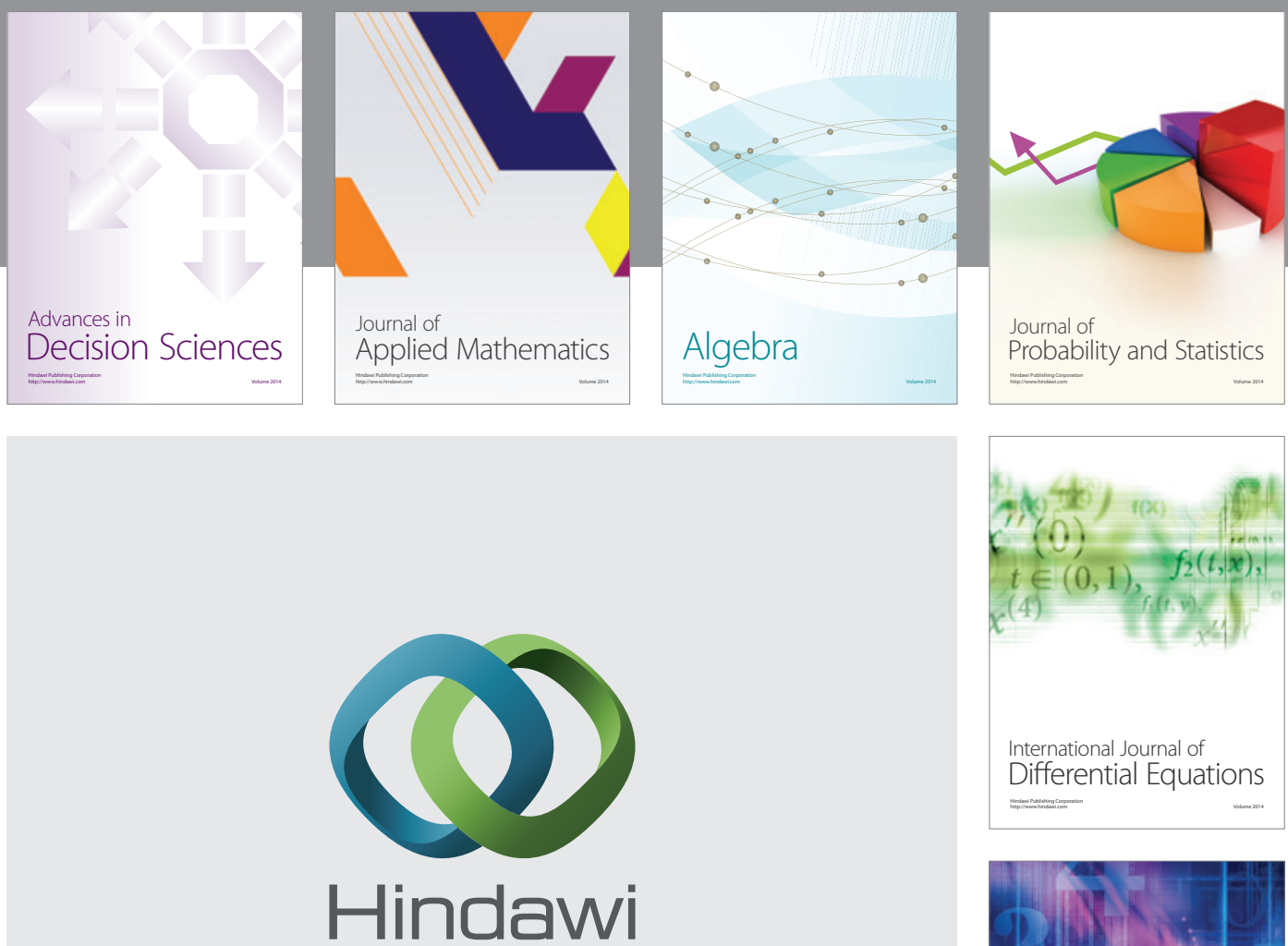

Submit your manuscripts at http://www.hindawi.com
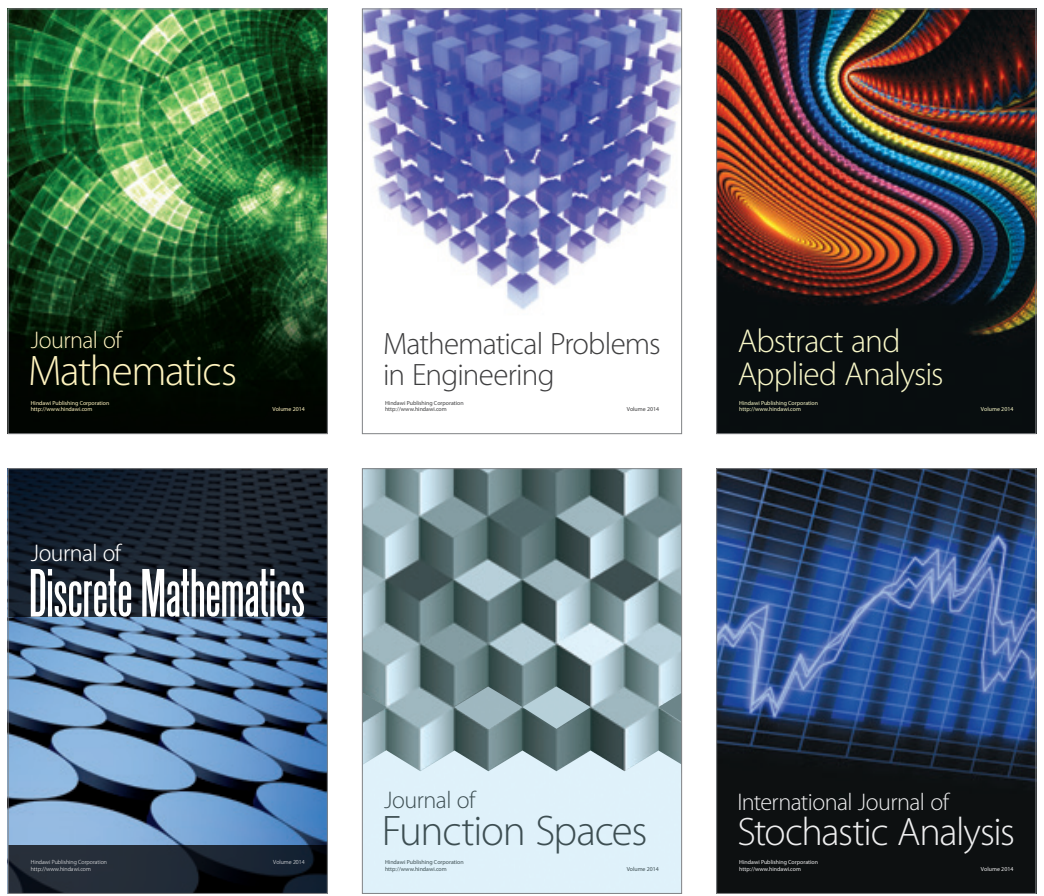

Journal of

Function Spaces

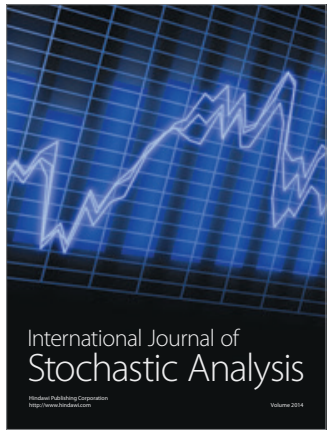

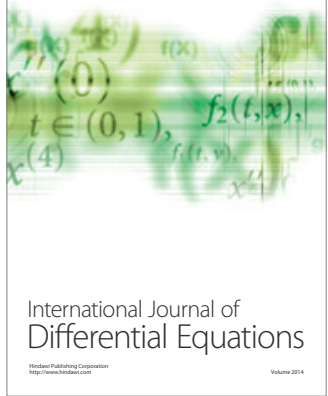
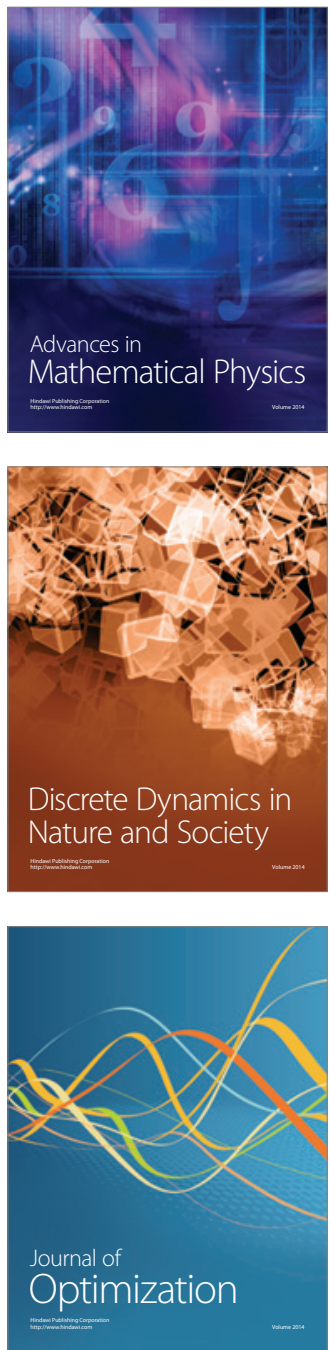